\title{
On the Origins of Truncated Lévy Flights
}

\author{
Annibal Figueiredo, Iram Gleria ${ }^{\mathrm{b}}$, Raul Matsushita ${ }^{\mathrm{c}}$, Sergio Da Silva ${ }^{\mathrm{d}, *}$ \\ ${ }^{a}$ Department of Physics, University of Brasilia, 70910-900 Brasilia DF, Brazil \\ ${ }^{\mathrm{b}}$ Department of Physics, Federal University of Alagoas, 57072-970 Maceio AL, Brazil \\ ${ }^{\mathrm{c}}$ Department of Statistics, University of Brasilia, 70910-900 Brasilia DF, Brazil \\ ${ }^{\mathrm{d} D e p a r t m e n t ~ o f ~ E c o n o m i c s, ~ F e d e r a l ~ U n i v e r s i t y ~ o f ~ R i o ~ G r a n d e ~ D o ~ S u l, ~ 90040-000 ~ P o r t o ~ A l e g r e ~ R S, ~}$ \\ Brazil
}

\begin{abstract}
We show that truncated Lévy flights appear due to the presence of particular features of autocorrelation in data. We present and analyze 'physical' reasons sufficient to ensure the scaling power laws and sluggish convergence associated with truncated Lévy flights. Our approach is exemplified with currency data for the British pound and Chinese yuan against the US dollar. We further compare these examples with a simulated Lorentzian distribution.
\end{abstract}

PACS: $05.40 .+\mathrm{j} ; 02.50 .-\mathrm{r}$

Keywords: Truncated Lévy Flights; Foreign Exchange Rates; Autocorrelation; Lorentzian Distribution

\section{Introduction}

Financial asset prices are unlikely to follow Gaussian distributions [1]. High peaks and fat tails are pervasive in financial data. Even a biased Gaussian random walk [2] cannot account for these 'anomalies'. Extreme events like Black Monday are virtually impossible in a biased random walk, where the probability of more than five standard deviations is essentially zero. Yet Black Monday is more than 34 standard deviations [3].

Early attempts to replace Gaussian distributions include the suggestion of a stable Lévy distribution to model cotton prices [4]. But though leptokurtosis can be accounted for by Lévy distributions, these have never been established in mainstream finance. One reason is related to their property of infinite variance. The number of data points of realworld financial series is always finite, and so it is the variance.

To remedy such a deficiency, a truncated Lévy distribution has been put forward [5, 6]. The truncated Lévy flight (TLF) aims at modeling financial series through a non-stable distribution which features non-normal scaling power laws and finite variance. The TLF is thus likely to model financial data. That has been shown for the S\&P500 index [6] and other stock indices $[7,8,9]$, as well as foreign exchange rates [10].

The TLF is a stochastic process defined for independent variables. And real-world financial data often present autocorrelations. However, for short-range autocorrelated data

\footnotetext{
* Corresponding author.

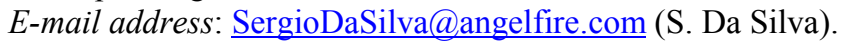


the observed statistical properties are frequently similar to those of random aggregative variables picked from the original time series [11]. This letter warns that that is not always the case.

Another contribution of this letter to literature is to show that an autocorrelation function, even if it is at its noise level, can lead to the scaling laws compatible with the presence of a TLF. What is more, we demonstrate how the autocorrelation acts as a friction causing the ultraslow convergence to the Gaussian regime, which is a typical feature of the TLF. We also show that random and non-random aggregative variables present distinct statistical properties, regardless of the autocorrelation. We exemplify our approach using data from the dollar prices of the British pound and Chinese yuan. We then contrast our results with a simulated Lorentzian, which is a special case of the Lévy distribution. This will allow us to compare real-world data fitted by a TLF with those of a 'true' TLF.

The structure of the letter is as follows. Section 2 briefly describes the TLF and puts forward 'physical' reasons for the sluggish convergence and power laws that are characteristic of TLFs. Section 3 presents an example of the relevance of the TLF to realworld exchange rates, namely the dollar prices of the pound and yuan. Our examples are then compared with a simulation of the Lorentzian distribution in Section 4. Section 5 concludes.

\section{Truncated Lévy flights and autocorrelation}

For a start, let us consider the ordinary Lévy distribution which is symmetric, i.e.

$$
P_{L}(S, \Delta t)=\frac{1}{\pi} \int_{0}^{\infty} \exp \left(-\gamma \Delta t q^{\alpha}\right) \cos (q S) d q,
$$

where $S=S_{\Delta t}=\sum_{i=1}^{\Delta t} x_{i}$, and $x_{1}, \ldots, x_{\Delta t}$ is a sequence of random variables. The process is then truncated $[5,6,8,10,11]$ according to

$$
P_{\Delta t}(S)= \begin{cases}c P_{L}(S, \Delta t), & |S| \leq l \\ 0, & |S|>l\end{cases}
$$

for some threshold $l$ and constant $c$. Distribution (2) is a TLF. It approaches equilibrium for the aggregative variables $S_{\Delta t}$ in accordance with the central limit theorem. When $\Delta t \rightarrow 1$ the process can be described by a stable Lévy probability density function (PDF).

Here we focus on the properties of the TLF of slow convergence to the Gaussian regime [5] and on the power law scaling in the probability of return to the origin [6]. Other features of the TLF are discussed elsewhere [11].

The probability of return to the origin is given by

$$
P_{\Delta t}(0)=\frac{\Gamma(1 / \alpha)}{\pi \alpha(\gamma \Delta t)^{1 / \alpha}} \Rightarrow \log P_{\Delta t}(0)=\log \left(\frac{\Gamma(1 / \alpha)}{\pi \alpha \gamma^{1 / \alpha}}\right)+(-1 / \alpha) \log \Delta t
$$

A straight line of slope $-1 / \alpha$ emerges in a plot of $\log P_{\Delta t}(0)$ versus $\log \Delta t$ in the time window for which the Lévy regime holds. The slope then asymptotically approaches -0.5 which is the value that corresponds to the Gaussian equilibrium.

Stable Lévy PDFs are self-similar [11]. To usefully compare the distributions for increasing values of $\Delta t$, scaled variables are taken, i.e. 


$$
\begin{aligned}
& S_{\Delta t}=\Delta t^{-1 / \alpha} S_{1}, \\
& P_{1}\left(S_{\Delta t}\right)=\Delta t^{-1 / \alpha} P_{\Delta t}\left(S_{1}\right)
\end{aligned}
$$

A given set of data can then be made to collapse onto the $\Delta t=1$ distribution, at least for the central region of the PDF. But there are departures from the Lévy PDF as far as the tails are concerned. Data points fall short of the ones of the Lévy which means that second moments are finite. For this reason, the process shows two regimes, a Lévy and a Gaussian. These are separated by a crossover time $\Delta t_{x}$ and are governed by distinct statistical properties.

Now we put forward possible 'physical' reasons for the appearance of the TLF in a time series. First consider 'central variables' defined as

$$
S_{\Delta t}^{\prime}=S_{\Delta t}-<S_{\Delta t}>
$$

and 'reduced variables' as

$$
\bar{S}_{\Delta t}=S_{\Delta t}^{\prime} / \sigma_{\Delta t}, \quad \sigma_{\Delta t}=\sqrt{<S_{\Delta t}^{2}>-<S_{\Delta t}>^{2}}
$$

So we take identically distributed (but not independent) variables.

For the characteristic function $\varphi(q)$ of a process with finite variance we have [12]

$$
\varphi(q)=e^{-q^{2}(1+w(q)) / 2}
$$

where $w(q)$ is a function such that $w(0)=0$. We state the following.

Definition 1. A stable process occurs in the interval $\left[\Delta t_{1}, \Delta t_{2}\right]$ if

$$
w_{\Delta t}(q)=u(q), \quad \forall \Delta t_{1} \leq \Delta t \leq \Delta t_{2}
$$

It is worth noting that Definition 1 does not turn the PDFs stable. Yet results that interest most are those with processes whose distributions are not stable. But Definition 1 does imply that the characteristic function is constant in the interval $\left[\Delta t_{1}, \Delta t_{2}\right]$, i.e.

$$
\bar{\varphi}_{\Delta t}(q)=\bar{\varphi}(q), \quad \Delta t_{1} \leq \Delta t \leq \Delta t_{2}
$$

where $\bar{\varphi}_{\Delta t}(q)$ is the characteristic function of $\bar{S}_{\Delta t}$ (and $\bar{\varphi}(q)$ is that of $\bar{S}_{1}$ ). Thus the reduced variables $\bar{S}_{\Delta t}$ have the same PDFs for $\Delta t \in\left[\Delta t_{1}, \Delta t_{2}\right]$ although their distribution laws $f\left(x_{i}\right)$ are not stable. For a process to be stable in the sense above and, at the same time, for the probabilistic laws $f(x)$ to be unstable, autocorrelation between the variables $x_{i}$ must be present.

If a process is independent, we have $\varphi_{\Delta t}(q)=\varphi^{\Delta t}(q)$ for the aggregative variables. By using the reduced variables we obtain

$$
\varphi_{S_{\Delta t}}(q) \rightarrow \varphi_{S_{\Delta t}}(q / \sqrt{\Delta t})=e^{-q^{2}(1+w(q / \sqrt{\Delta t})) / 2}
$$

The central limit theorem follows because [12]

$$
\Delta t \rightarrow \infty \Rightarrow e^{-q^{2}(1+w(0)) / 2}=e^{-q^{2} / 2}
$$

From (11) we realize that (9) is satisfied only if there is a friction preventing the convergence $w_{\Delta t}(q) \rightarrow 0$ as $\Delta t \rightarrow \infty$. And such a friction is precisely the one found in correlated processes.

Now let us consider an interesting property of a stable process concerning the scaling in $P\left(S_{\Delta t}=<S_{\Delta t}>\right)$. If $\widetilde{\varphi}(q)$ is the characteristic function of our central variables (5), then we have 


$$
P\left(S_{\Delta t}=<S_{\Delta t}>\right)=P\left(S_{\Delta t}^{\prime}=0\right)=\int_{-\infty}^{\infty} \widetilde{\varphi}(q) d q=\int_{-\infty}^{\infty} \bar{\varphi}_{\Delta t}\left(\sigma_{\Delta t} q\right) d q
$$

By performing the transformation $q^{\prime}=\sigma_{\Delta t} q$ we get

$$
P\left(S_{\Delta t}^{\prime}=0\right)=\frac{1}{\sigma_{\Delta t}} \int_{-\infty}^{\infty} \bar{\varphi}_{\Delta t}\left(q^{\prime}\right) d q^{\prime}
$$

Since the process is stable in $\Delta t_{1} \leq \Delta t \leq \Delta t_{2}$, the integral above equals a constant $A$. Thus

$$
P\left(S_{\Delta t}^{\prime}=0\right)=P\left(S_{\Delta t}=<S_{\Delta t}>\right)=\frac{A}{\sigma_{\Delta t}}
$$

So the probability of return to the origin scales with the inverse of the standard deviation $\sigma_{\Delta t}$ in the interval of stability $\left[\Delta t_{1}, \Delta t_{2}\right]$. And if the standard deviation itself scales as a power law of type

then we have

$$
\sigma_{\Delta t}=C \Delta t^{1 / \alpha}, \quad \Delta t_{1} \leq \Delta t \leq \Delta t_{2}
$$

$$
P(0)=\left(\frac{A}{C}\right) \frac{1}{\Delta t^{1 / \alpha}} \Rightarrow \log P(0)=\log \left(\frac{A}{C}\right)+\left(-\frac{1}{\alpha}\right) \log \Delta t
$$

Note that Eq. (16) is similar to (3) if

$$
\left(\frac{\Gamma(1 / \alpha)}{\pi \alpha \gamma^{1 / \alpha}}\right)=\frac{A}{C}
$$

Here the relevance of the autocorrelation should be stressed. For independent and identically distributed (IID) variables, $\alpha=1 / 2$ in (15). For correlated processes $\alpha \neq 1 / 2$.

Now suppose that $w_{\Delta t}(q)$ varies very slowly in the interval $\left[\Delta t_{1}, \Delta t_{2}\right]$. Such a process can be dubbed 'quasi-stable'. Here a relation similar to (16) holds, i.e.

$$
P(0)=\left(\frac{A_{\Delta t}}{\sigma_{\Delta t}}\right), A_{\Delta t}=\int_{-\infty}^{\infty} \bar{\varphi}_{\Delta t}\left(q^{\prime}\right) d q^{\prime}=\int_{-\infty}^{\infty} e^{-q^{2}\left(1+w_{\Delta t}(q)\right) / 2} d q
$$

Since $w_{\Delta t}$ is almost constant in the interval $\left[\Delta t_{1}, \Delta t_{2}\right]$, then $A_{\Delta t}$ varies very slowly. If the standard deviation $\sigma_{\Delta t}$ obeys scaling law (15), then we have a relation similar to (17) with $A_{\Delta t}$ replacing $A$.

Moreover if $\alpha \leq 2$ then the probability of return to the origin follows a scaling similar to that of a stable distribution and is characterized by characteristic function $\varphi(q)=e^{-k|q|^{\alpha}}$.

So the central region of the distribution follows a Lévy process even if the distributions $f(x)$ themselves are not either stable, independent, or generated by a Lévy stochastic process.

For a quasi-stable process there exists a time horizon in which $w_{\Delta t}(q)$ approaches zero very slowly because the autocorrelation acts as a friction. That is precisely the reason why the ultraslow convergence associated with the TLF emerges. However, as $w_{\Delta t}(q) \approx 0$ the characteristic function gets closer to the Gaussian, i.e.

$$
\bar{\varphi}(q) \approx e^{-q^{2} / 2}, \quad\left(w_{\Delta t} \approx 0, \Delta t \geq \Delta t_{x}\right)
$$

in which case $P(0)$ rescales as predicted for a normal distribution.

It is worth noting that none of the results above hold true if $w_{\Delta t}$ is not sluggish. It is thus implied that there exists particular types of autocorrelation associated with TLFs. But 
we have no means to learn whether a particular kind of autocorrelation is at first compatible with a TLF.

\section{Example}

Our approach can be illustrated with reference to the dollar prices of the British pound and Chinese yuan. Such data were taken from the Federal Reserve website at http: //www.federalreserve.gov/releases/H10/hist/ (Table 1). These exchange rates were collected by the Federal Reserve Bank of New York from a sample of market participants. They are noon buying rates in New York from cable transfers payable in these currencies. As standard, here we ignore 'holes' from weekends and holidays; analysis thus focuses on trading days. We also take returns $Z$ rather than raw data, i.e.

$$
Z_{\Delta t}(t)=Y(t+\Delta t)-Y(t)
$$

where $Y(t)$ is a rate at day $t$. Fig. 1 displays the logarithm of the PDFs of currency returns. Increases in time horizons range from $\Delta t=1,2$, and 5 trading days (a week) to 240 trading days (a year). Incidentally note that the aggregative variables $S_{\Delta t}$ are equivalent to $Z_{\Delta t}(t)$.

We showed elsewhere [10] that the TLF is likely to be pervasive among daily time series of currency returns in dollar terms, the yuan being among the exceptions. We have chosen the pound and yuan as typical examples because the pound/dollar returns are possibly described by a TLF but the same cannot be said as far as the yuan/dollar returns are concerned. Here our aim is to illustrate what makes it more likely for a process to be described by a TLF by focusing on autocorrelations.

Fig. 2 shows a log-log plot of $P(0)$ against $\Delta t$. Power laws emerge for the currencies in the time window of $1 \leq \Delta t \leq 100$. These are at first consistent with the presence of a TLF.

By plugging the slope value of $-1 / \alpha$, we get $\gamma$ from (3). By using (4), the data can then collapse onto the $\Delta t=1$ distribution (Fig. 3). Fig. 3 suggests that a TLF is unlikely to model the yuan/dollar returns.

Table 2 displays the parameters $\alpha$ and $\gamma$ for the currencies. Parameter $\alpha \approx 1.8$ for the pound/dollar returns is well inside the Lévy regime which stops at 2. Parameter $\alpha \approx 5$ for the yuan/dollar returns is far outside the Lévy regime.

Both the pound and yuan exhibit scaling in their second moments (Fig. 4). From the curve for the pound we get the following fitting:

$$
\begin{aligned}
& \sigma_{\mathrm{S}_{\Delta t}^{\mathrm{o}}}^{\mathrm{o}}=0.009641091 \Delta t^{0.549272} \\
& \frac{1}{\alpha}=0.549272 \Rightarrow \alpha=1.820591619
\end{aligned}
$$

where $\Delta t$ ranges from 1 to 50. As expected from our discussion in Section 2, parameter $\alpha$ is in good agreement with that in Table 2. Indeed both the presence of power laws in the second moment and a quasi-stable function $w(q)$ lead to the power law in the probability of return to the origin. Expression (21) is theoretically given by

$$
\sigma_{S_{\Delta t}}=\sigma \sqrt{\Delta t+\sum_{i, j}^{\Delta t} \operatorname{corr}\left(x_{i}, x_{j}\right)}
$$

It is straightforward to conclude from (22) that $\sum \operatorname{corr}\left(x_{i}, x_{j}\right)$ is the responsible for the particular curve followed by the original process in Fig. 4. Fig. 5 adds up the 
autocorrelations of $S_{\Delta t}^{o}$ to the curve of the process for 'randomized variables' $S_{\Delta t}^{R}$. The latter is a sum of variables taken randomly from a time series for $S_{1}=Z_{1}(t)$ which in turn is expected to follow an IID process. Note that the new adjusted curve matches that of $S_{\Delta t}^{o}$. The original process of the aggregative variables $S_{\Delta t}^{o}$ is shown in Fig. 4 together with $S_{\Delta t}^{R}$. Fig. 4 suggests that $S_{\Delta t}^{o}$ and $S_{\Delta t}^{R}$ follow distinct scaling laws. That is at first unexpected for processes with the autocorrelation function at its noise level. Short-range autocorrelation is usually considered as a sufficient condition for $S_{\Delta t}^{o}$ and $S_{\Delta t}^{R}$ to share the same statistical properties. Yet that might not be always true.

Indeed for the pound the autocorrelation is somehow short range (Fig. 6). But short-range autocorrelated processes usually present a characteristic time which is not seen in Fig. 6. The autocorrelation starts out at 0.06 (maximum at 1) and goes on very low throughout. Despite the fact that the autocorrelation remains at its noise level, it is still responsible for the scaling law leading to the TLF for the pound. There seems to be no difference in the autocorrelations of the pound and yuan. However, as we will see below the yuan returns are not described by the TLF.

Fig. 7 displays the behavior of function $w(q)$ for both currency returns. Function $w(q)$ can be rewritten as $w(q)=w_{R}+i w_{i}$. And this expression can be further expanded to yield

$$
\left\{\begin{array}{l}
w_{R}=-\frac{1}{12}\left(\mu^{4}-3\right) q^{2}-\left(\frac{\mu^{4}}{24}+\frac{\mu^{3}}{36}-\frac{1}{12}+\frac{\mu^{6}}{360}\right) q^{4}+\ldots \\
w_{R}=-\frac{1}{12}(K) q^{2}+O\left(q^{4}\right)
\end{array}\right.
$$

where $\mu^{\Delta t}=\sum_{i=1}^{N}\left[\frac{x_{i}-<x>}{\sigma}\right]^{\Delta t}$, and $K=\mu^{4}-3$ is the kurtosis. The leading term of $w_{R}$ (kurtosis) is shown in Fig. 7 for the currencies to both $S_{\Delta t}^{o}$ and $S_{\Delta t}^{R}$. For the pound we can see that the process is quasi-stable in the sense discussed above. Note that $S_{\Delta t}^{o}$ is sluggish and $S_{\Delta t}^{R}$ converges faster to the Gaussian regime. That is not unexpected since $S_{\Delta t}^{R}$ follows the behavior of an IID process. Here the kurtosis measures the convergence speed of the process. As far as the yuan is concerned, Fig. 4 shows that $S_{\Delta t}^{o}$ and $S_{\Delta t}^{R}$ alike follow the curve of an IID process. Thus, since both $S_{\Delta t}^{o}$ and $S_{\Delta t}^{R}$ follow a stochastic process, they exhibit the same convergence speed and $w(q)$ cannot show in Fig. 7 the quasi-stability necessary for the TLF to emerge.

It is worth noting that we can also take into account the imaginary part of $w(q)$, whose leading terms involve the skewness of the process

$$
\left\{\begin{array}{l}
w_{I}=\frac{\mu^{3}}{3} q+\left(\frac{1}{6} \mu^{3}-\frac{\mu^{5}}{60}\right) q^{3}+\frac{1}{12}\left(\mu^{3}-\frac{\mu^{5}}{10}-\frac{\mu^{3} \mu^{4}}{6}\right) q^{5}+O\left(q^{7}\right) \\
w_{I}=\frac{1}{3}(J) q+\frac{1}{6}(J) q^{3}+O\left(q^{5}\right)
\end{array}\right.
$$

Rather than focusing on the kurtosis, the leading terms in the expansion of the norm $\sqrt{\left|w_{I}\right|^{2}+\left|w_{R}\right|^{2}}$ can alternatively be seen as a measure of the distance to the Gaussian regime. 
So despite the fact that the yuan/dollar returns show scaling in the second moment (Fig. 4), that feature by itself is not enough for the TLF to be present (Fig. 7 and Table 2).

As seen (Fig. 6) there is no sharp difference in the autocorrelations for the pound and yuan. But now we have learned that, unlike the pound, both $S_{\Delta t}^{o}$ and $S_{\Delta t}^{R}$ for the yuan are governed by the same statistical laws. Though it remains to be seen which particularities in these 'noise' autocorrelations are leading to such distinct behaviors.

To spice up our discussion we report another intriguing property of the yuan/dollar returns. Fig. 8 displays an IFS-clumpiness test [13] where white noise generates a picture in which dots fill it uniformly. Correlated noise and perhaps chaotic data present localized clumps. Idiosyncratically a fractal known as the Sierpinsky triangle emerges [14, 15]. And the same test for the pound gives a patternless cloud of dots (not shown). Perhaps the noise autocorrelation function has something to do with the nested behavior in Fig. 8. But we leave such a conjecture for future research.

\section{Comparison with a simulated Lorentzian distribution}

This section presents a simulation of a stable Lévy stochastic process. Our motivation is the following. The quasi-stability and relation (15) suffice for a TLF to fit the modal region of data. Nevertheless none of these are necessary. Our simulation will lead to a function $w(q)$ that is not quasi-stable. What is more, the second moment scales with $n^{1 / 2}$, which is typical of an IID process. But $P(0)$ scales with the parameter of the Lévy distribution generating our simulated data.

We consider the particular case of a Student's $t$ distribution [11]

$$
P\left(z_{\Delta t}\right)=\frac{C_{\Delta t}}{\left(1+z_{\Delta t}^{2} / \Delta t\right)^{(\Delta t+1) / 2}}
$$

where $C_{\Delta t}=\frac{\Gamma[(\Delta t+1) / 2]}{\sqrt{\pi \Delta t} \Gamma(\Delta t / 2)}$ is part of stochastic process

$$
z_{\Delta t}=\frac{h \sqrt{\Delta t}}{\sqrt{u_{1}^{2}+\cdots u_{\Delta t}^{2}}}
$$

obtained from independent variables $u_{i}$ and $h$, each with normal density, zero mean and variance equaling the unity. Note that $\Gamma(\Delta t)$ is the Gamma function. For $\Delta t=1, P(z)$ collapses to a Lorentzian distribution, i.e. the Lévy distribution with $\alpha=1$ and $\beta=0$. For $\Delta t>1$, the Lorentzian is the only stable case of the Student's $t$ distributions. As a general rule, $P(z)$ becomes the Gaussian distribution with finite moments of order $k$ when $\Delta t \rightarrow \infty$ and $k<\Delta t$. Such distributions have been considered by some [16] as an alternative to the Gaussian.

Fig. 9 displays our numerical simulation for the Lorentzian, where the $\log$ of $P(Z)$ is plotted against $Z$. Fig. 10 shows that the second moment scales with $\Delta t^{1 / 2}$, as expected for a Gaussian process. And $P(0)$ scales with $\alpha=1$ (Fig. 11) and (as also expected) can collapse onto the $\Delta t=1$ distribution (Fig. 12).

By comparing the example of the Lorentzian with that of the pound (Fig. 6 and 13), we realize that both processes present autocorrelations at their noise level. However, the speed of convergence of the pound is much slower than that of the Lorentzian. This can be seen in the behavior of function $w(q)$ (Fig. 14). Thus the autocorrelation does not act as a 
friction for the Lorentzian, and is not responsible for the scaling in $P(0)$. Unlike the pound and yuan cases, our simulation with the Lorentzian shows that the scaling laws typical of a TLF appear for reasons other than autocorrelation in data.

As seen at the end in Section 4, we do not know which are the particularities in noise autocorrelations that can lead to distinct behaviors. Our analysis of a simulated Lorentzian distribution suggests that such a problem is far from being trivial. Indeed, scaling laws typical of a TLF may appear for reasons other than autocorrelation for the Lorentzian.

\section{Conclusion}

The TLF is a stochastic process defined for independent variables. However, realworld financial data are often autocorrelated. To justify the use of the TLF, short-range autocorrelated data are usually treated as independent after their characteristic time has elapsed.

This letter shows that the definition of a TLF need not rely on the assumption of independence. Indeed, we demonstrate that an autocorrelation function even at its noise level can also lead to scaling laws that are compatible with the presence of the TLF. What is more, we show how the autocorrelation acts as a friction causing the ultraslow convergence to the Gaussian regime, which is a typical feature of the TLF. Our discussion is illustrated with the pound and yuan returns in dollar terms and then with a simulated Lorentzian distribution.

Thus the main characteristics of the TLF, such as power law scaling in the probability of return to the origin and the slow speed of termalization, are due to particularities of the autocorrelation in data.

A problem is that the specific nature of these particularities is currently unknown. Analysis of a simulated Lorentzian distribution shows that such a problem is far from being trivial. Indeed, our simulation with the Lorentzian show that the scaling laws typical of a TLF appear for reasons other than autocorrelation in data.

\section{Acknowledgements}

We are grateful to Aline De Almeida for computer assistance and to Pushpa Rathie for discussions. 


\section{References}

[1] A. Lo, A. C. MacKinlay, Stock market prices do not follow random walks: evidence from a simple specification test, Rev. Fin. Stud. 1 (1988) 41-66.

[2] L. Bachelier, Theorie de la speculation (PhD thesis in mathematics), Ann. Sci. Ecole Norm. Sup. III-17 (1900) 21-86.

[3] H. E. Stanley, L. A. N. Amaral, X. Gabaix, P. Gopikrishnan, V. Plerou, Similarities and differences between physics and economics, Physica A 299 (2001) 1-15.

[4] B. B. Mandelbrot, The variation of certain speculative prices, J. Business 36 (1963) 394-419.

[5] R. N. Mantegna, H. E. Stanley, Stochastic process with ultraslow convergence to a Gaussian: the truncated Lévy flight, Phys. Rev. Lett. 73 (1994) 2946-2949.

[6] R. N. Mantegna, H. E. Stanley, Scaling behavior in the dynamics of an economic index, Nature 376 (1995) 46-49.

[7] L. C. Miranda, R. Riera, Truncated Lévy walks and an emerging market economic index, Physica A 297 (2001) 509-520.

[8] I. Gleria, R. Matsushita, S. Da Silva, Scaling power laws in the Sao Paulo Stock Exchange, Econom. Bull. 7 (2002) 1-12.

[9] J. A. Skjeltorp, Scaling in the Norwegian stock market, Physica 283 (2001), 486-525.

[10] A. Figueiredo, I. Gleria, R. Matsushita, S. Da Silva, Autocorrelation as a source of truncated Lévy flights in foreign exchange rates, Physica A, forthcoming.

[11] R. Mantegna, H. E. Stanley, An Introduction to Econophysics, Correlations and Complexity in Finance, Cambridge University Press, Cambridge, 2000.

[12] D. Dugué, Oeuvres de Paul Lévy, Vol. III: Eléments Aléatoires, Gauthiers-Villars, Paris, 1976.

[13] J. C. Sprott, Chaos data analyzer: the professional version 2.1, American Institute of Physics, 1995.

[14] S. Da Silva, A. Figueiredo, There is something about the yuan/dollar rate, preprint available at http://www.angelfire.com/id/SergioDaSilva/yuanannibal.pdf.

[15] R. Matsushita, I. Gleria, A. Figueiredo, S. Da Silva, Fractal structure in the Chinese yuan/US dollar rate, preprint available at http://www.angelfire.com/id/SergioDaSilva/matsushita.pdf.

[16] R. C. Blattberg, N. J. Gonedes, A comparison of the stable and Student distributions as statistical model for stock prices, J. Business 47 (1974) 244-280. 
Table 1

Description of data sets

\begin{tabular}{l|l|c|c}
\hline Country & Currency & Time Period & Data Points \\
\hline Britain & Pound & 4Jan71 - 10Jan03 & 8033 \\
\hline China & Yuan & 2Jan81 - 10Jan03 & 5472 \\
\hline
\end{tabular}

Table 2

Parameters $\alpha$ and $\gamma$ for the currencies in Table 1

\begin{tabular}{l|c|c}
\hline Currency & $\alpha$ & $\gamma$ \\
\hline Pound & 1.75110 & .000092423 \\
\hline Yuan & 4.97749 & $3.9307 \mathrm{E}-13$ \\
\hline
\end{tabular}



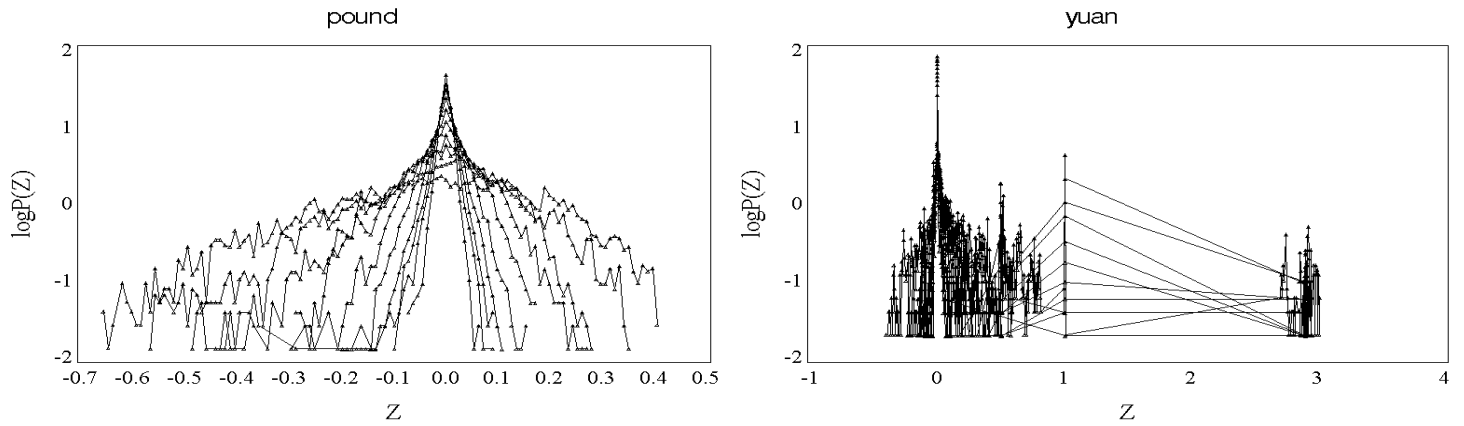

Fig. 1. Probability density functions of the pound and yuan returns observed at time intervals $\Delta t$ which range from 1 to 240 trading days. As $\Delta t$ is increased, a spreading of the probability distribution characteristic of any random walk is observed.
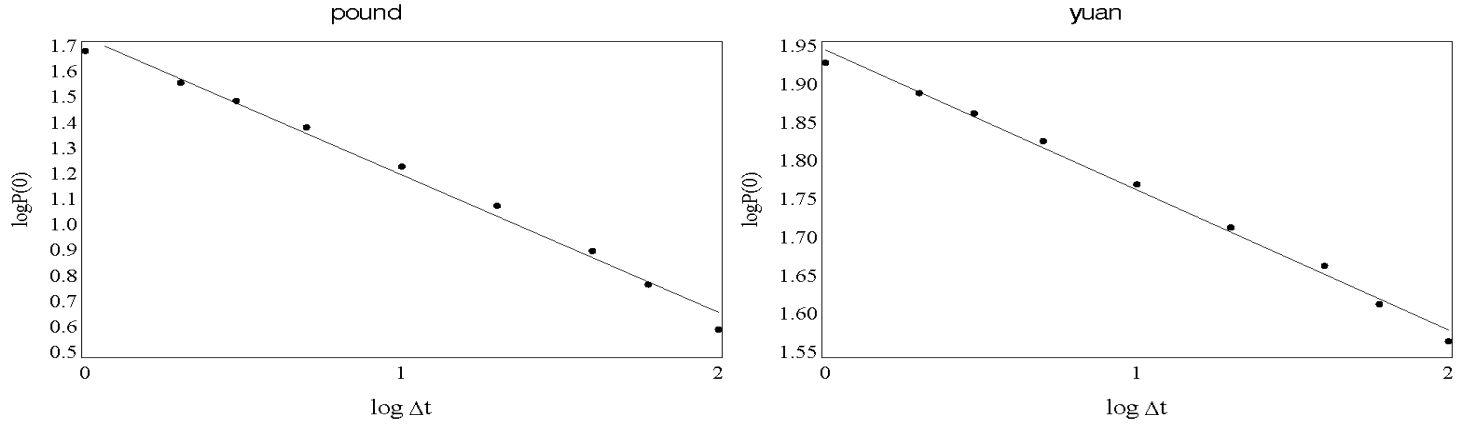

Fig 2. Log-log plot of the probability of return to the origin $P(0)$ against the time lag $\Delta t$ for the pound and yuan returns in dollars. Power laws emerge in the time window of $1 \leq \Delta t \leq 100$. This non-Gaussian scaling is at first consistent with the presence of a TLF.
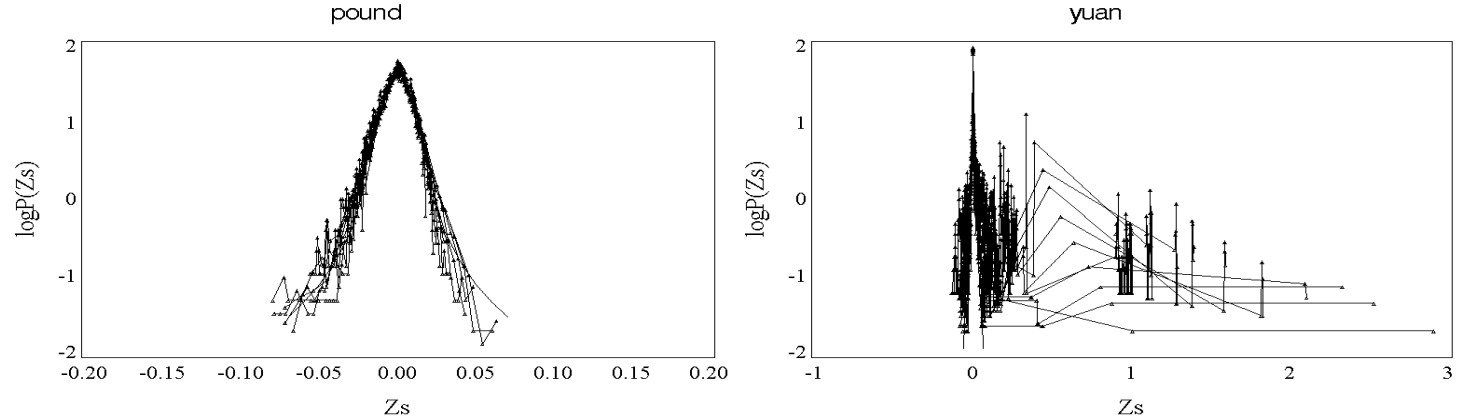

Fig. 3. The same PDFs as in Fig. 1 but now plotted in scaled units $P(Z)$. Given the scaling index $\alpha$ for a currency (Table 2), all the data are made to collapse onto a $\Delta t=1$ distribution. The pound seems to adjust well, but the same cannot be said as far as the yuan is concerned. 

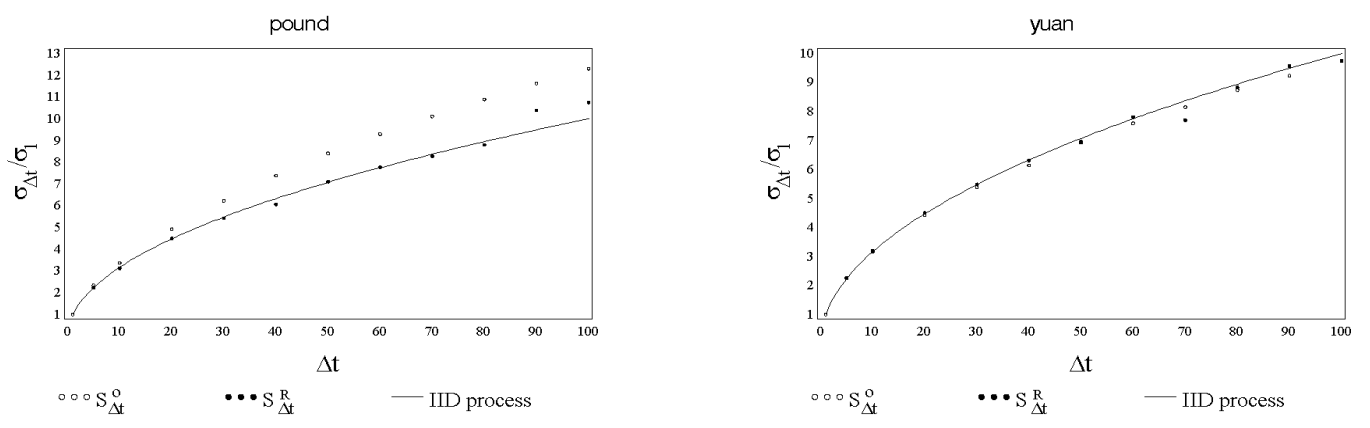

Fig. 4. Plot of the scaled second moment versus $\Delta t$ for both the original process $\left(S_{\Delta t}^{o}\right)$ and the randomized process $\left(S_{\Delta t}^{R}\right)$. The curve of an IID process $\sigma_{\Delta t}=\sigma \sqrt{\Delta t}$ is shown for comparison. For the yuan/dollar returns, the fitting line of the original process $S_{\Delta t}^{o}$ is $-3.13338+0.49122 \log (\Delta t)$ and that of the randomized process $S_{\Delta t}^{R}$ is $-3.13352+0.49305 \log (\Delta t)$; thus both processes follow the curve of an IID process.

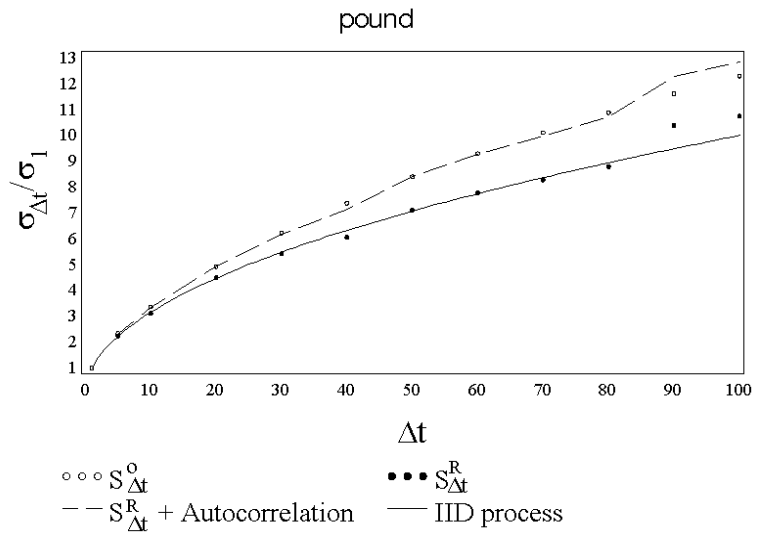

Fig. 5. The role of the autocorrelation for the pound/dollar returns. The dotted curve is $S_{\Delta t}^{R}$ added up to the autocorrelations of $S_{\Delta t}^{o}$.
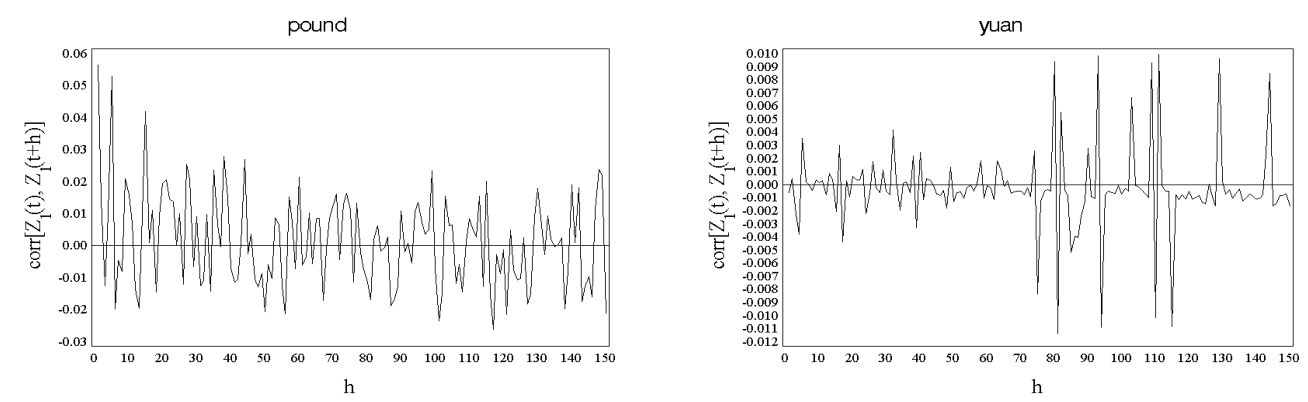

Fig. 6. Autocorrelation function $\operatorname{corr}\left[Z_{1}(t), Z_{1}(t+h)\right]$ for the pound and yuan returns in dollar terms. 

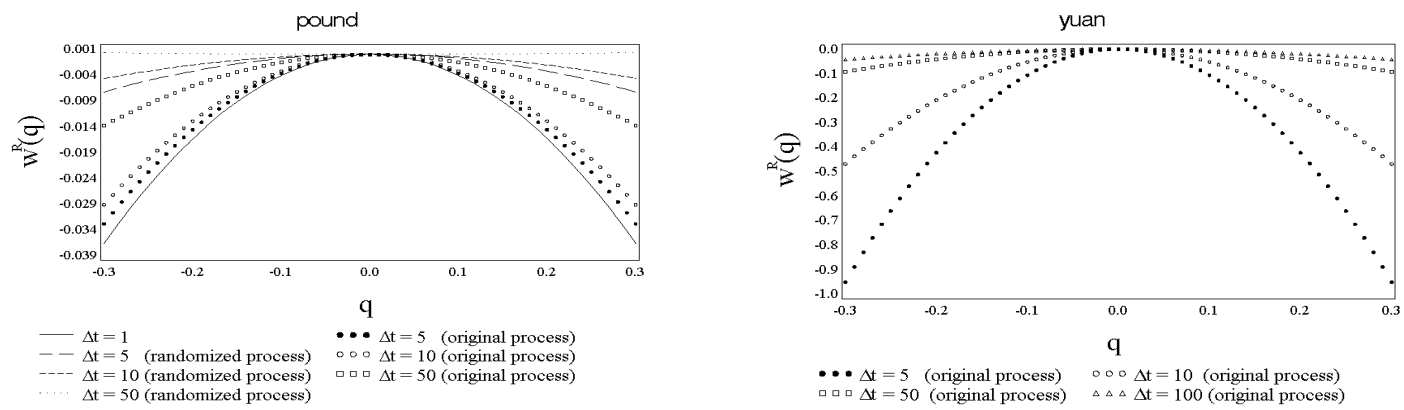

Fig. 7. Behaviour of the kurtosis for the pound and yuan returns. For the pound we can see that the process is quasi-stable. Note that $S_{\Delta t}^{o}$ is sluggish and $S_{\Delta t}^{R}$ converges faster to the Gaussian regime. For the yuan, since both $S_{\Delta t}^{o}$ and $S_{\Delta t}^{R}$ follow a stochastic process, $w(q)$ does not exhibit the quasi-stability necessary for the TLF to emerge.

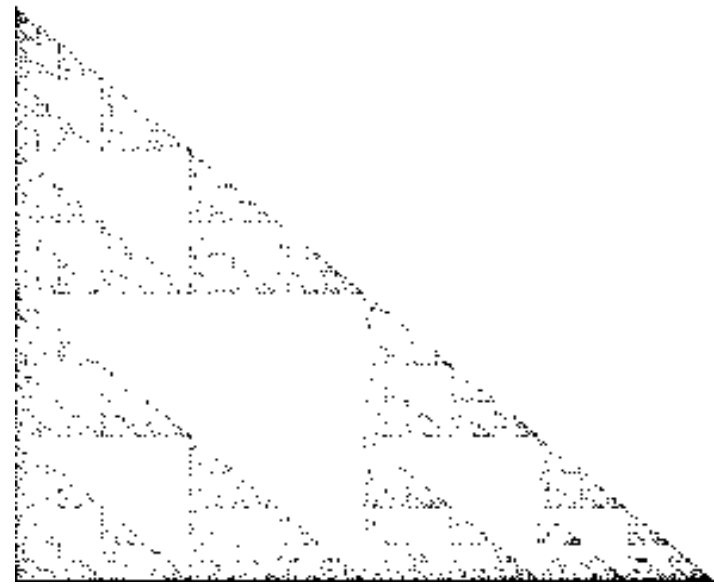

Fig. 8. IFS clumpiness test for the price changes of the yuan/dollar rate.

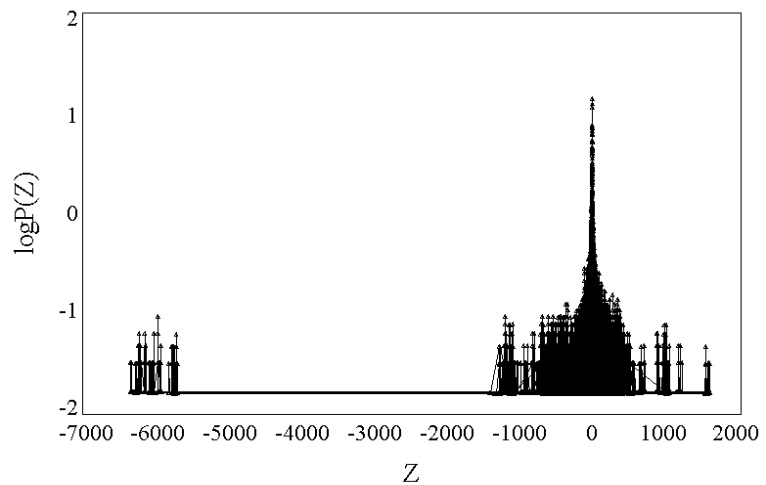

Fig. 9. Logarithm of $P(Z)$ versus $Z$ for the simulated Lorentzian process. 


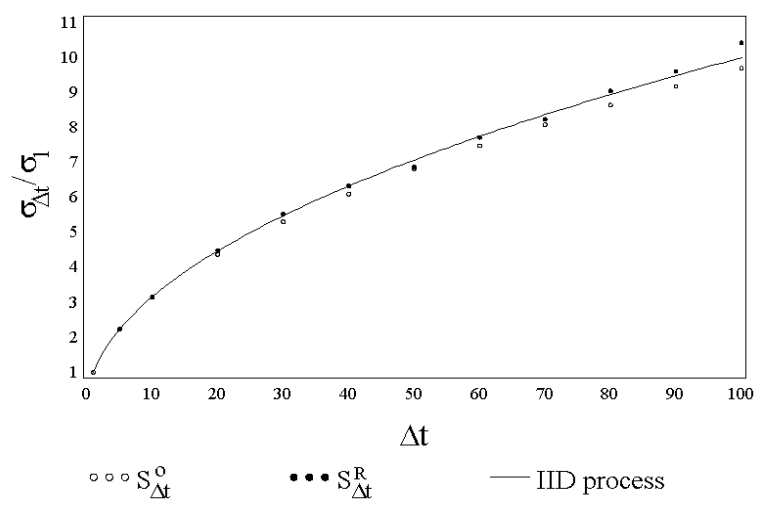

Fig. 10. Curve followed by the second moment for the Lorentzian.

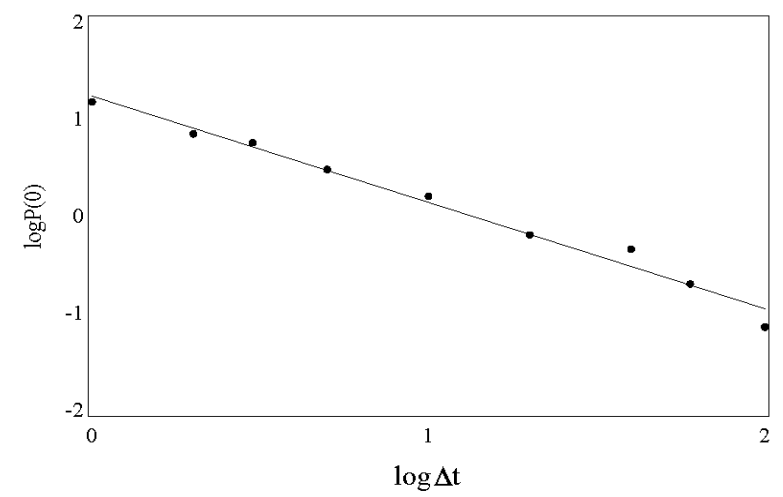

Fig. 11. Scaling in $P(0)$ for the Lorentzian.

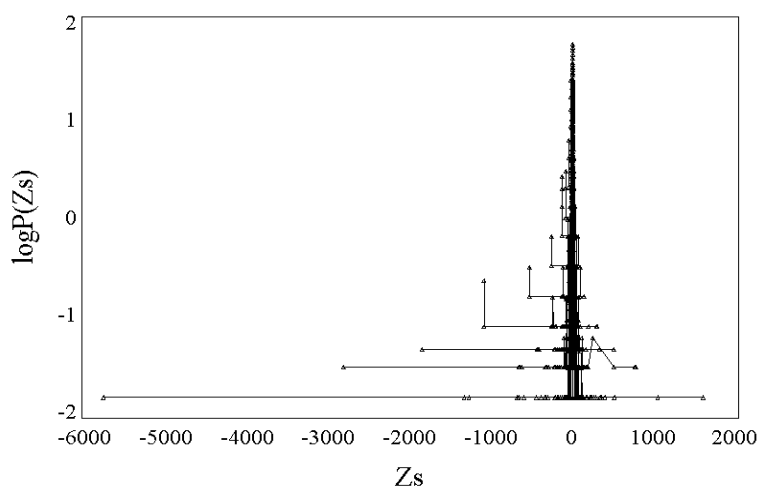

Fig. 12. Collapse onto the $\Delta t=1$ distribution for the Lorentzian. 


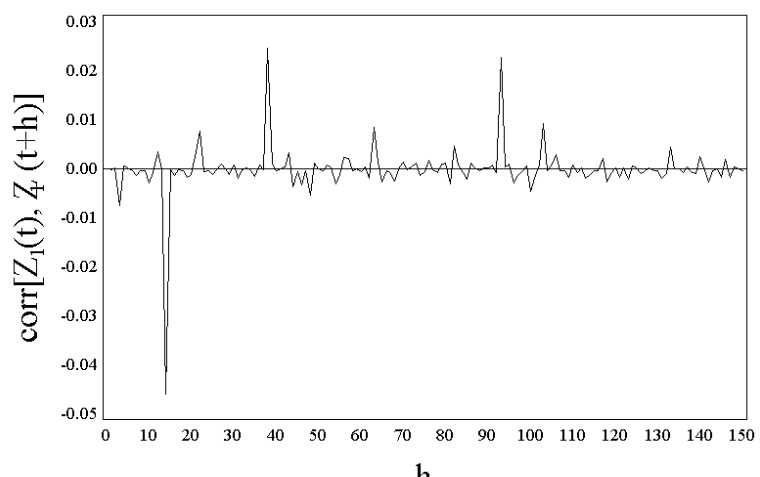

Fig. 13. Autocorrelation for our simulation of the Lorentzian.

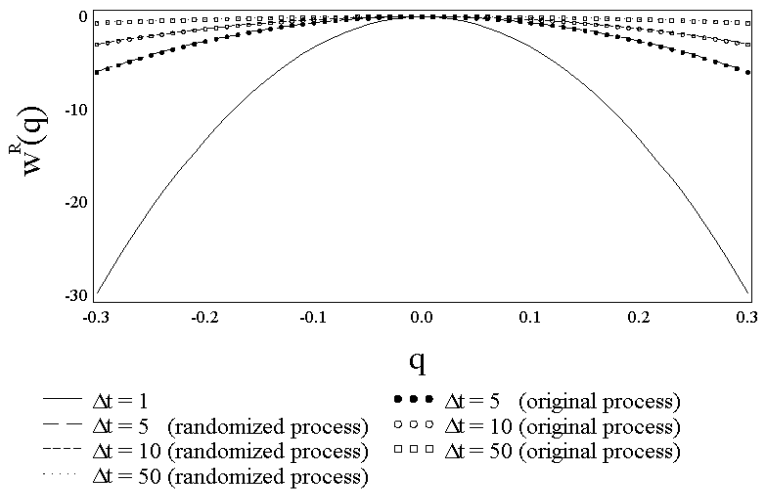

Fig. 14. Behavior of $w(q)$ for the Lorentzian distribution. 